\title{
Keeping track of time: evidence for episodic-like memory in great apes
}

\author{
Gema Martin-Ordas · Daniel Haun • \\ Fernando Colmenares $\cdot$ Josep Call
}

Received: 28 April 2009/Revised: 10 September 2009/Accepted: 14 September 2009/Published online: 27 September 2009

(C) The Author(s) 2009. This article is published with open access at Springerlink.com

\begin{abstract}
Episodic memory, as defined by Tulving, can be described in terms of behavioural elements (what, where and when information) but it is also accompained by an awareness of one's past (chronesthesia) and a subjective conscious experience (autonoetic awareness). Recent experiments have shown that corvids and rodents recall the where, what and when of an event. This capability has been called episodic-like memory because it only fulfils the behavioural criteria for episodic memory. We tested seven chimpanzees, three orangutans and two bonobos of various ages by adapting two paradigms, originally developed by Clayton and colleagues to test scrub jays. In Experiment 1, subjects were fed preferred but perishable food (frozen juice) and less preferred but non-perishable food (grape). After the food items were hidden, subjects could choose one of them either after $5 \mathrm{~min}$ or $1 \mathrm{~h}$. The frozen juice was still available after $5 \mathrm{~min}$ but melted after $1 \mathrm{~h}$ and became unobtainable. Apes chose the frozen juice significantly more after $5 \mathrm{~min}$ and the grape after $1 \mathrm{~h}$. In Experiment 2, subjects faced two baiting events happening at different times, yet they formed an integrated memory for the location and time of the baiting event for particular food items. We also included a memory task that required no temporal encoding. Our results showed that apes remember in an integrated fashion what, where and when (i.e., how long ago) an event happened; that is, apes distinguished between different events in which the same food items
\end{abstract}

G. Martin-Ordas $(\bowtie) \cdot$ D. Haun · J. Call

Max Planck Institute for Evolutionary Anthropology,

Deutscher Platz 6, 04103 Leipzig, Germany

e-mail: ordas@eva.mpg.de

G. Martin-Ordas · F. Colmenares

Departamento de Psicobiología de la Facultad de Psicología de la Universidad Complutense de Madrid, Madrid, Spain were hidden in different places at different times. The temporal control of their choices was not dependent on the familiarity of the platforms where the food was hidden. Chimpanzees' and bonobos' performance in the temporal encoding task was age-dependent, following an inverted U-shaped distribution. The age had no effect on the performance of the subjects in the task that required no temporal encoding.

Keywords Episodic-like memory $\cdot$ Age $\cdot$ Great apes

\section{Introduction}

A crucial component of cognition is memory. Memory is made up of a number of different and inter-related systems that are defined, among other features, by how we access them or the type of information encoded (Miyashita 2004, Squire 1992). In the study of memory, one of the most influential distinctions is between semantic and episodic memory systems (Tulving 1972). Semantic memory refers to the retention of factual or propositional information that may (or may not) be personal or autobiographical. By contrast, episodic memory stores personal past events. Tulving $(1983,2005)$ defined episodic memory in terms of its content and the subjective experiences that accompany it (autonoetic awareness). The content of episodic memory refers to information about what, where and when a specific event occurred. The subjective experiences that accompany episodic recall require the re-experience of the past event and it also involves in oneself who travels back to a point in time and, therefore, is able to have a subjective sense of past, present and future time (chronesthesia). The definition in terms of these phenomenological constructs makes it extremely difficult, if not impossible, to 
demonstrate this type of memory in nonverbal species because there are no agreed upon non-linguistic behavioural markers of conscious experience.

In recent years, several studies with animals have dealt with this phenomenon from different approaches. Clayton and colleagues have shown that scrub jays flexibly integrated memories for the what, where and when of an event (Clayton and Dickinson 1998, 1999; Clayton et al. 2001, 2003b). Other bird and mammal species have also been shown to possess such type of memory [rodents: Babb and Crystal 2005, 2006; Ergorul and Eichenbaum 2004; Ferkin et al. 2008; Roberts et al. 2008; Zhou and Crystal 2009; birds: Henderson et al. 2006 (when and where, but not what), Zinkivskay et al. 2009; Bird et al. 2003; Hampton et al. 2005; Mckenzie et al. 2005; Skov-Rackette et al. 2006 for negative results in rats, pigeons and monkeys]. This capability fulfils Tulving's behavioural criteria for episodic memory (Tulving 1972) and is referred to as episodic-like memory rather than episodic memory because it does not assess subjective experiences that accompany conscious recollection in humans (Clayton and Dickinson 1998, Clayton et al. 2003a). However, Suddendorf and Busby (2003) pointed out that this type of memory should be more properly called www-memory. They argued that one could know what happened, where and when (e.g., know when you were born) without actually being able to remember the event. Likewise, personal memories are not always accurate. Therefore, remembering a personal past event does not necessarily involve remembering the when and the where of the past event.

Zentall et al. (2001) (see also Morris and Frey 1997) have suggested that in order to study episodic memory, the test should be unexpected because the episodic information should be encoded automatically; that is, episodic memory experiments should be based on trial-unique learning. Therefore, one weakness in Clayton's experimental approach is that it does not measure memory for unique experiences because they require training. Clayton et al. (2003b) (see also Salwiczek et al. 2008) have argued that learning about the properties of the food items during the training in their experiment with the scrub jays could be viewed as the acquisition of semantic information that is applicable to different events in a flexible way. Therefore, Clayton et al. (2003b) (see also Salwiczek et al. 2008) proposed that searching for recovery is controlled by semantic information (perishability of the cached food items) but also by episodic-like recall.

The trial-unique learning paradigm has been successfully implemented in rodents (rats: Kart-Teke et al. 2006; mice: Dere et al. 2005) and birds (Zentall et al. 2001). KartTeke et al. (2006) (see also Dere et al. 2005) presented rats with a three trials object exploration task in which memory for what (object recognition), where (location of the objects) and when (temporal order for the presentation of the objects) were combined. In the first sample trial, subjects explore four copies of a novel object. After a time delay, subjects are presented with a second sample trial, identical to the first, except that four novel objects were present, which were arranged in a different spatial configuration. After another delay, the subjects received a test trial identical to the second sample trial, except that two copies of the object from sample trial 1 ("old familiar" objects) and two copies of the object known from sample trial 2 ("recent familiar" objects) were present and one of the "old familiar" objects was shifted to a location in which it was not encountered during the sample trial 1 . The results from these experiments showed that rats were sensitive not only to the location of the objects, but also to the temporal order in which they were presented. These results led the authors to conclude that rats integrated what, where and when an event happened.

Zentall et al. (2001) used a delayed-matching to the sample task with pigeons in which they were required to remember whether or not they performed a particular action in the past. The results from this experiment showed that pigeons were able to report which action they performed in the past. However, as pointed out by Crystal (2009), episodic memory is defined as a long-term memory system and the unexpected question experiment carried out by Zentall et al. (2001) only deals with short-time delays between the encoding event and the experimental question.

An alternative approach suggested by Eacott et al. (2005) challenged the main role of the "when" component in episodic-like memory. Following Friedman (1993) (see Suddendorf and Busby 2003 and Suddendorf and Corballis 2007), they argue that human episodic memory is poor in recalling the timing of an event and, therefore, "when" serves exclusively as a marker to distinguish an event from another event. Hence, they proposed that episodic-like memory in animals should involve the recall of the what, where and which (Eacott et al. 2005). Eacott et al. (2005) carried out a study in which the rats had to explore two different objects placed in certain spatial configuration in a first context based on their tendency of exploring novel objects. Next, these same objects were placed in a different configuration in a second context. Following this episode but prior to their re-introduction into one of the two previously experienced contexts, the rats were exposed to one of the objects in a different place. This experience would enhance rats' propensity to seek out the other object when they were returned to one of the previous contexts. However, they could only look for the other object, if they could remember where the objects were located in a particular context during the initial event. Rats' success in this task allowed the authors to conclude that rats recollected the what, where and which of a past episode. 
Compared to rodents and birds, non-human primates have received relatively little attention in episodic-like memory research. Schwartz et al. (2002) investigated whether a gorilla could remember who did what. In the training phase, the gorilla had to learn to associate five types of food and their English words with five wooden cards in which a picture of each food was represented. The gorilla also had to associate two trainers with their respective names. In the experimental condition, the two trainers were present, although only one of them gave him one of the food items. Some time later (either $10 \mathrm{~min}$ or $24 \mathrm{~h}$ ) the gorilla was provided with a set of seven cards, five for the different types of food and two for the two trainers. He was asked what he ate and who gave him the food in that particular episode. The gorilla was able to hand over the card that represented the type of food that was given to him and the card with the name of the trainer who had given him the food after the delay (Schwartz et al. 2002). However, it is still an open question whether the gorilla recalled the event or simply chose the cards that were more familiar to him (Schwartz 2005; Schwartz et al. 2005). In a free recall experiment, a chimpanzee correctly indicated to the caretakers where a specific food item was hidden using a lexigram keyboard up to $16 \mathrm{~h}$ after observing baiting events (Menzel 2005). Note that one possible alternative explanation for the chimpanzee's performance is spatial semantic memory; that is, the chimpanzee may have updated her memory about spatial landmarks without recalling the food-hiding event. Additionally, none of these studies have shown that great apes can remember when the event occurred. The sole attempt to evaluate www-memory in non-human primates has been done with rhesus macaques (Hampton et al. 2005). In their study, Hampton and colleagues adapted the paradigm implemented by Clayton and Dickinson (1998) with the jays and they showed that macaques were unable to remember the when component of an event. Recently, Hoffman et al. 2009 examined working memory (Baddeley 2000) for what, where and when information in rhesus monkeys using a new paradigm based on a computer task and found positive evidence for episodic-like memory. However, note that episodic memory has been described as a long-term memory system and that working memory is considered as a short-term memory, therefore, dissociated from episodic memory in humans. Hoffman et al. (2009) argued that rhesus macaques integrated the what, where and when. However, their subjects were asked to remember these three elements in separate occasions, raising doubts about the integration of the components. Therefore, we consider that this procedure is vulnerable to criticism and, in consequence, stronger evidence for episodic-like memory in non-human primates is still needed.
In the studies presented here, we investigated whether great apes encode information about what is hidden where and when (www-memory) by adapting two paradigms that have been successfully used with scrub jays (Clayton and Dickinson 1998; Clayton et al. 2001). Additionally, we investigated the developmental trajectory of this ability by studying apes of various ages. We were particularly interested in knowing whether performance showed the same age-dependent distribution seen in humans. Evidence from several studies on episodic memory in humans describes a symmetrical inverted U-shaped pattern of rising in childhood (3-4 years old) and declining in ageing (by the age of 25-30 years it begins a steady decline through adulthood) (see Tulving and Craik 2000; Craik and Salthouse 2000, 2008 for reviews). We contrasted the wwwmemory task with a memory task that required no temporal encoding and showed no dramatic changes in ability across the ages in humans (Tulving and Craik 2000; Craik and Salthouse 2000, 2008). We tested chimpanzees, bonobos and orangutans because they represent our closest and most distant great ape relatives, respectively. This will allow us to make inferences about the evolution of this cognitive skill. It has also been argued that episodic memory and future planning depend on common processes and recent experimental studies have shown evidence for planning in chimpanzees, bonobos and orangutans (Mulcahy and Call 2006; Dufour and Sterck 2008; Osvath and Osvath 2008; but see Suddendorf 2006, Suddendorf et al. 2009 for a critical review). Therefore, positive evidence for episodiclike memory in these three species will support the idea that these two phenomena depend on the same processes.

\section{Experiment 1: content of memories}

In this experiment, we investigated if subjects can remember when and where two types of food were hidden. We presented the subjects with a platform in which three baiting places were available. The experimenter baited two of the three locations. One location contained a favourite but perishable food (frozen juice) and the other location contained a less preferred but non-perishable food (grape). The third location remained empty. After the food was hidden, subjects were allowed to choose one of these items either after a 5-min or 1-h retention interval (RI). After $1 \mathrm{~h}$, the frozen juice melted and became unobtainable, but it was still edible if chosen after $5 \mathrm{~min}$. If apes can remember when and where the two types of food were hidden, they should show a preference for the frozen juice on the 5-min trials because they should expect the frozen juice to be edible. However, this preference should be reversed on the 1-h trials if they can remember that the frozen juice was 
hidden a relatively long time ago and, therefore, would not be obtainable.

Methods

\section{Subjects}

We tested two bonobos (Pan paniscus), seven chimpanzees (Pan troglodytes) and three orangutans (Pongo pygmaeus) housed at the Wolfgang Köhler Primate Research Centre in the Leipzig Zoo (Germany). There were four males and eight females with ages ranging from 6 to 31 years (Table 1). We tested subjects individually (except for Jahaga, who was tested accompanied by a male) and none were food- or water-deprived. Subjects had previously participated or were currently participating in other studies (e.g., tool use, planning), so they were all used to participate in tests.

\section{Apparatus and procedure}

The apparatus consisted of 36 different opaque containers and two plastic platforms $(70 \mathrm{~cm} \times 35 \mathrm{~cm})$. In one of the platforms, we drilled three holes and covered them with a plastic net. This net allowed the liquid to go through it and be collected under the platform inside a hidden cup.

\section{Procedure}

The experimenter (E) and the subject sat facing each other on either side of the Plexiglass partition with three equidistant circular holes (6 $\mathrm{cm}$ diameter) on its bottom part just above the platform (on E's side). E placed three containers on the platform about $30 \mathrm{~cm}$ apart in front of a vertical Plexiglass, showed one reward (pre-test) or two rewards (what, where and when memory test) to the subject and placed them under one/two of the containers, depending on test. There were three phases:

Memory ability pre-test In the pre-test, we tested individuals' general long-term memory. Subjects had to remember the location of a reward placed under one of three cups on a platform after 2-min, 1-, 2- and 24-h RIs. The $2 \mathrm{~min}, 1,2$ and $24 \mathrm{~h}$ trial designations refer to the length of the time that elapsed between baiting and letting the apes to choose one of the containers. Apes received a total of 12 trials, 3 per condition. We used slices of banana as rewards.

Food preference test Prior to the experiment, we established the apes' preference for two food items. We placed one piece of each of two foods on the platform. Food included grapes (approximately $3 \mathrm{~cm}$ long $\times 1.5 \mathrm{~cm}$ diameter), slices of banana (approximately $0.5 \mathrm{~cm}$ ) and frozen juice (approximately $3 \mathrm{~cm}$ long $\times 2.5 \mathrm{~cm}$ wide $\times 1.5 \mathrm{~cm}$ high). Various food combinations were used (i.e., banana vs. frozen juice, grapes vs. frozen juice) until each subject chose a given food, at least, five times over six trials. The food-locations were counterbalanced and apes could choose only once in each trial. We ranked frozen juice as the high-value food over banana for two chimpanzees and frozen juice as the high-value food over grapes for the rest of the subjects.

It is important to mention that the apes did not have any previous experience with the frozen juice that we used in

Table 1 Percentage of choices to the baited container (pre-test) and the frozen juice (experiments 1 and 2) for each subject. A direct comparison between conditions at the individual level in Experiment 1 is also shown

\begin{tabular}{|c|c|c|c|c|c|c|c|c|c|c|c|c|}
\hline \multirow[t]{2}{*}{ Name } & \multirow[t]{2}{*}{ Genus } & \multirow[t]{2}{*}{ Age } & \multicolumn{4}{|c|}{ Pre-test } & \multicolumn{4}{|c|}{ Experiment 1} & \multicolumn{2}{|c|}{ Experiment 2} \\
\hline & & & $2-\min$ & $1-\mathrm{h}$ & $2-h$ & 24-h & $5-\min$ & 1-h & $\chi^{2}$ & $p$ & 5 -min & 1-h \\
\hline Kuno & Pan paniscus & 11 & 100 & 100 & 100 & 66.66 & 72.22 & 27.78 & 7.11 & 0.018 & 66.66 & 16.67 \\
\hline Limbuko & Pan paniscus & 12 & 100 & 100 & 100 & 66.66 & 66.66 & 23.53 & 6.55 & 0.018 & 33.33 & 50 \\
\hline Alex & Pan troglodytes & 6 & 100 & 66.66 & 100 & 100 & 94.44 & 88.24 & 0.43 & NS & NT & NT \\
\hline Lome & Pan troglodytes & 6 & 100 & 66.66 & 100 & 100 & 58.88 & 31.25 & 0.35 & NS & NT & NT \\
\hline Alexandra & Pan troglodytes & 8 & 100 & 100 & 33.33 & 66 & 88.88 & 68.75 & 1.83 & NS & NT & NT \\
\hline Jahaga & Pan troglodytes & 14 & 100 & 100 & 100 & 100 & 88.88 & 33.34 & 11.68 & 0.002 & 50 & 0 \\
\hline Fifi & Pan troglodytes & 14 & 100 & 100 & 100 & 66.66 & 83.33 & 44.45 & 5.9 & 0.035 & 80 & 20 \\
\hline Dorien & Pan troglodytes & 27 & 100 & 100 & 100 & 100 & 60 & 50 & 0.33 & NS & NT & NT \\
\hline Fraukje & Pan troglodytes & 31 & 100 & 100 & 100 & 33.33 & 88.88 & 83.34 & 0.23 & NS & NT & NT \\
\hline Padana $^{\mathrm{a}}$ & Pongo pygmaeus & 10 & 100 & 100 & 100 & 66.66 & 100 & 77.23 & 0.58 & NS & 100 & 50 \\
\hline Dokana & Pongo pygmaeus & 18 & 100 & 100 & 33.33 & 100 & 94.44 & 88.24 & 0.43 & NS & NT & NT \\
\hline Pini & Pongo pygmaeus & 19 & 100 & 100 & 66.66 & 100 & 94.44 & 100 & 1.02 & NS & NT & NT \\
\hline
\end{tabular}

$N T$ not tested

${ }^{a}$ Padana was presented with Experiment 2 because she chose 4 times in row out of 6 the grape in the 1-h trial in the last round of Experiment 1 
our experiment. However, they have had experience with snow and in summer, as a part of their enrichment activities, they are sometimes provided with big pieces of ice cubes containing fruit.

What-where-when memory test There were three possible baiting places on the platform. One was baited with frozen juice, another with the less preferred food and the third site remained unbaited.

Subjects were presented with both 5-min and 1-h trials. The 5-min and 1-h designation refer to the length of the time that elapsed between baiting and letting the apes to choose one of the containers. On the 5-min trials, the frozen juice and the less preferred food were available, whereas on the 1-h trial the frozen juice melted and only the less preferred food was available. The order in which subjects were presented in the two types of trials was counterbalanced. Apes received a total of three 12-trials rounds. To provide a unique baiting location on every trial within each round for each ape, the three baiting sites were covered with three different containers each. Additionally, these containers were different for each trial. In each round and for each trial different baiting places were used and the three possible sites were counterbalanced.

\section{Analyses}

We videotaped all trials. Subject's choice was counted as the first box touched. We used Wilcoxon test to analyse whether individuals' performance in the memory ability pre-test differed from chance. We also used Wilcoxon test to assess whether subjects chose the frozen juice more often in the 5-min than in the 1-h condition. We used Pearson Chi-square to investigate subject's choices in the 5-min and 1-h trials at individual level. We analysed the effect of the age using the percentage of correct responses in the memory ability pre-test and the difference between the percentage of choices of the frozen juice at $5 \mathrm{~min}$ and $1 \mathrm{~h}$ in the what-where-when memory test. To assess the relation between performance and age, we fitted the models expressing performance as a quadratic function of age, i.e. performance $=c_{0}+c_{1} \times$ age $+c_{2} \times$ age $^{2}$. We used the 'glm' function provided by the R base package (R Development Core Team 2007) to fit the equation. Since the sample sizes were small and potentially included outliers, we used the quadratic term based on permutation (Adams and Anthony 1996; Manly 1997) to test levels of significance. We randomized performance across the subjects and measured the coefficient of the quadratic term $\left(c_{2}\right.$ see equation above) for the randomized data. Finally, we determined the $p$ value of the quadratic term as the proportion of permutations revealing an absolute coefficient being at least as large as the absolute coefficient of the original data. We used 1,000 permutations with the original data included as one permutation. Since we tested a nullhypothesis twice (no relation between age and performance), an error level adjustment was required. We achieved this using Fisher's Omnibus test. This procedure combines a number of $p$ values into a single $c^{2}$-distributed variable with degrees of freedom equalling twice the number of $p$ values (Haccou and Meelis 1994). All statistical tests were two-tailed.

\section{Results}

\section{Pre-test}

Overall apes remembered the location of the food after each RI above the chance levels in the memory ability pretest (Wilcoxon test: $z=3.12, p=0.002, n=12$ in all intervals, Fig. 1) and there was no change in performance across ages $\left(r_{\mathrm{s}}=0.08, p=0.41, n=12\right)$.

\section{What-where-when memory test}

If apes can remember when and where the two types of food were hidden, they should show a preference for the frozen juice on the 5-min trials (frozen juice is still available) but this preference should be reversed on the 1-h trials (the frozen juice is no longer available). Subjects chose the frozen juice significantly more often after the 5-min interval than after the 1-h interval (Wilcoxon test: $z=2.55, \quad p=0.011, n=12$; Fig. 2). Their choices remained unchanged during testing (Friedman tests: $5-\min -\chi^{2}=0.16, \quad n=12, \quad p=0.92 ; 1-\mathrm{h}-\chi^{2}=0.42$, $n=12, p=0.8$ ). Individual analyses showed that 4 out of 12 subjects chose the frozen juice significantly more often than the grape after the short RI and the grape after the long RI (Table 1). Since subjects rarely selected the empty

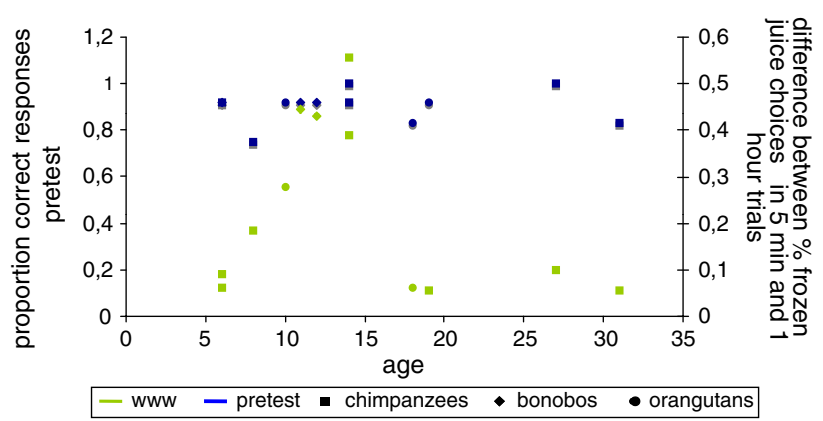

Fig. 1 Proportion of correct responses in the pre-test and wwwmemory (Experiment 1) as a function of age. Proportion of correct responses in the www-memory test refers to the difference between percentage choice of the frozen juice at $5 \mathrm{~min}$ and $1 \mathrm{~h}$ 


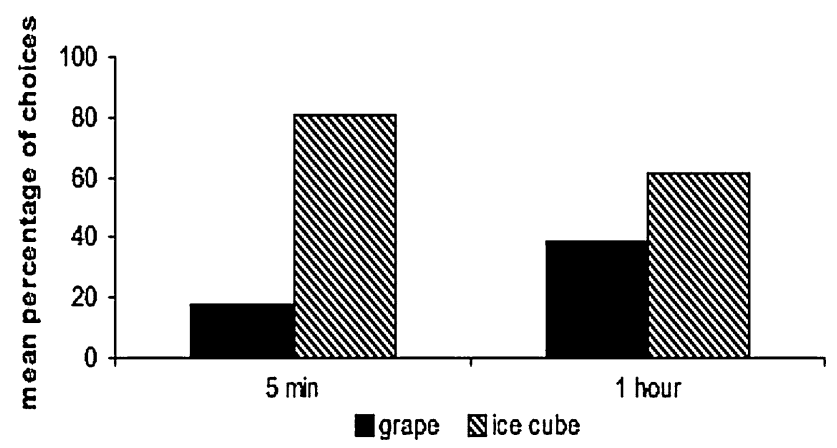

Fig. 2 Mean percentage of choices of frozen juice and grape in the 5-min and 1-h trials

boxes in the pre-test and test (pre-test: less than $12 \%$ of the trials, test: less than $3 \%$ of the trials), this suggests that their choices of the less preferred food after 1-h delay were not simply due to memory lapses. Unlike general long-term memory in the pre-test (permutation test: $c_{2}=-0.013$, $p=0.375, n=12$, Figure 1), the performance on the www-memory test revealed an inverted U-shaped trend as a function of age (permutation test: $c_{2}=-0.142$, $p=0.0682, n=12$, Fig. 1). Focusing solely on members of the genus Pan (chimpanzees and bonobos) confirmed this result (permutation test: $c_{2}=-0.026, p=0.002$, $n=9$ ). Subjects younger than 7 and older than 18 years of age showed a much lower performance than adolescents and young adults.

\section{Discussion}

Subjects' performance can only be explained by the recall of three types of information: what types of food (frozen juice and grapes) were hidden, where they were hidden (in which box) and when (5 min or $1 \mathrm{~h}$ ago) they were hidden. In terms of purely behavioural criteria, our results provide evidence for episodic-like memory in great apes.

Additionally, apes' performance in the www-memory task showed an inverted U-shaped curve in which middleage subjects performed better than the youngest and oldest subjects. In contrast, there was no age effect in the pre-test in which no temporal information was needed to be encoded. During the pre-test, subjects were capable of remembering the location of the food reward after $24 \mathrm{~h}$ had elapsed since baiting. In fact, the performance in the 24-h delay condition was as good as that after a 2-min delay condition. Interestingly, longitudinal and cross-sectional studies in human memory have reported an inverted U-shaped curve as a function of age for episodic memory and no age effect for long-term memory (Bialystok and Craik 2006 for a review)

\section{Experiment 2: structure of memories}

Experiment 1 established that apes can remember the what, where and when of an event, although this does not indicate the structure of the memories that support subjects' choices (Clayton and Dickinson 1999; Clayton et al. 2001, 2003a). If the three components occurred in an integrated representation, interrogating any episodic memory for any feature of the event will retrieve the other two features. One possible structure is a what-where-when. The presence of the tables when subjects have to make their choice activates "where" representations of the food, which also activates "what" representations of the food and "when" representations encoding information about how long ago the baiting event took place. The content and temporal information is integrated in the memory of the baiting event through the "where" representation of the baited places. By structuring their memories in what-where-when fashion, subjects would be able to distinguish between similar events that happen at different times. An alternative structure is where-what-when. This memory structure allows subjects to retrieve "what" representations that activate the "where" and "when" representations, however, with no direct association between "where" and "when". Therefore, this structure does not explicitly encode information about how long ago the baiting event happened in a particular location. As a consequence, an animal using this representation would be unable to discriminate two episodes sharing the same "what" because the "where" and the "when" components are not directly connected. We examined these possibilities in Experiment 2 by administering those subjects that performed best in Experiment 1 with another task (Clayton et al. 2001) in which subjects witnessed two baiting events separated by $1 \mathrm{~h}$. Five minutes after the second baiting event, the apes were allowed to choose two times in succession, either first from the table baited $1 \mathrm{~h}$ ago and then from the table baited $5 \mathrm{~min}$ before or vice versa. If apes encoded the baiting events into coherent integrated what-where-when structures, they should preferentially choose the frozen juice in the table baited last but the grape in the table baited first. Otherwise, their preferences should be indistinguishable for the two tables. For this experiment, we tested the successful subjects only from the previous experiment because their choices were based on how long ago the baiting event took place. We included one of the orangutans because in the last round of Experiment 1 she significantly reversed her choices $\left(\chi^{2}=6, p=0.014\right.$; see table 1 for more details). Subjects' success was essential to determine whether they could distinguish between different episodes. The nature of this experiment was confirmatory. 
Methods

\section{Subjects}

We tested two bonobos (Pan paniscus), two chimpanzees (Pan troglodytes) and one orangutan (Pongo pygmaeus) (see table 1 for more details). There were two males and three females with ages ranging from 10 to 14 years of age. We tested subjects individually (except for Jahaga, who was tested accompanied by a male) and none were food- or water-deprived.

\section{Apparatus and procedure}

The apparatus consisted of 36 different opaque containers and two plastic platforms $(70 \mathrm{~cm} \times 35 \mathrm{~cm})$. In the platforms, we drilled three holes and covered them with a plastic net. This net allowed liquid to go through it and be collected under the platform inside a hidden cup.

\section{Procedure}

We presented subjects with two platforms located in two different testing cages. The general procedure in every trial consisted of two different baiting episodes happening at different times: E entered the testing cage and baited two of the containers with frozen juice and food, the third one remained empty. After $1 \mathrm{~h}, \mathrm{E}$ came back to the testing cage and baited the other platform following exactly the same procedure as before. After $5 \mathrm{~min}, \mathrm{E}$ went into the testing cage and let the subject to choose one of the containers from one of the two platforms. All the subjects received 5-min and 1-h trials. In the 5-min trial apes choose first in the platform baited 5 min before and next in the platform baited $1 \mathrm{~h}$ before, whereas in the 1-h trial the order was reversed. The order of the $5 \mathrm{~min}$ and 1 -h trials and the baited sites on the platform were counterbalanced across subjects. Subjects received a total of 6 trials, 3 per condition.

\section{Analyses}

We used Wilcoxon test to assess whether subjects chose the frozen juice more often in the 5-min than in the 1-h condition. The statistical test was one-tailed given that we had an a priori prediction.

Results

Subjects chose the frozen juice significantly more often in the table baited last compared to the table baited first (Wilcoxon test: $z=1.79, p=0.037,1$-tailed, $n=5$, Table 1).
Discussion

The results of this experiment confirmed the findings from the first experiment, that is, subjects' choices are mediated by the temporal information. To solve this task the apes cannot rely on discrimination by relative familiarity because both platforms are always present during the entire trial. It is possible, then, that the order in which the platforms were baited and the platform in which they chose first are the variables that they need to take into account to make their choices. Therefore, subjects have to remember the two baiting events not only in terms of their time, but also in terms of the location of the food even though they both involved the same food items. Additionally, Experiment 2 demonstrates that the encoded information about the what, where and when of an event occurred in an integrated representation. A where-what-when memory cannot support these results because the presence of either table should have retrieved the "what" representation of frozen juice cubes and grapes. These food item representations should have also activated representations for both $5 \mathrm{~min}$ and $1 \mathrm{~h}$, RIs and for the four locations of the food (frozen juice cubes and grapes). If apes' memories were structured in a where-what-when fashion, they would have confused the time at which the two baiting events took place and, therefore, they would neither have been able to use their perishability knowledge to choose the frozen juice after $5 \mathrm{~min}$ nor would have avoided choosing the frozen juice baited $1 \mathrm{~h}$ earlier. In contrast, a what-where-when structure supports our findings because the "where" representation triggers both the "what" but also the "when" representation of the baiting event. Apes chose more often the grape in the platform baited first because the frozen juice hidden in that platform was baited $1 \mathrm{~h}$ earlier and, therefore, had melted. Likewise, they chose more often the frozen juice in the platform baited last because the frozen juice was hidden 5 min ago and was, therefore, still edible. The binding between what-where-when allows subjects to discriminate between different episodes that share common features.

\section{General discussion}

Similar to other species (Babb and Crystal 2005, 2006; Clayton and Dickinson 1998, Clayton et al. 2001, 2003b; Dere et al. 2005; Ergorul and Eichenbaum 2004; Ferkin et al. 2008; Henderson et al. 2006; Roberts et al. 2008; Zinkivskay et al. 2009), great apes encoded information about what was hidden, where and when (how long ago) in an integrated fashion. Additionally, subjects' performance in the www-memory task showed an age-dependent inverted U-shaped curve. In contrast, no age effect was 
observed in a memory task that required no encoding of temporal information.

Friedman (1993) described three types of information that humans (but also animals) could process: temporal locations, which refer to when an event took place within a time pattern; temporal distances, implying how long ago an event occurred and before-after relations or order of events. Friedman (2007) has suggested that sense of time in humans depends on inferring when the event must have happened by using general knowledge of conventional (e.g., days of the weeks, months), natural or personal time patterns. However, humans also have impressions of the age of the events. Research on memory for the past events in humans has shown that there is no evidence that events are coded by the times of their occurrence or that memory is temporally organised (Friedman 1993, 2004). Recently, Roberts et al. (2008) have suggested that in www-memory studies (like the one presented here), subjects could be using elapsed time by means of circadian timers, accumulators or the strength of a memory trace as a cue to make their choices rather than remembering when an event happened (i.e. mentally travel in time to an event of the past). Particularly important in the experiments presented here is the fact that great apes took into account temporal information to choose a food item. However, the mechanism that apes used to keep track of time and the nature of the temporal representation of the past events remains undetermined. There are at least two possibilities that do not involve explicit temporal encoding in episodic-like memory: forgetting (Friedman 1993, 2001) and familiarity (Kort et al. 2005).

We consider that the most direct form of forgetting (i.e. subjects forget the location of the food items) can be ruled out because apes chose empty boxes quite rarely. Alternatively, it could be possible that subjects used forgetting as a cue to make their choices. If apes use the strength of the baiting memory to locate the episode, they should also show some evidence of forgetting for other aspects of the content of the memory (what or where) at longer RIs. The results of the pre-test demonstrate that apes possessed an accurate memory for the location of the food, that is, they were as accurate at remembering where an item was hidden after $24 \mathrm{~h}$ as they were after $2 \mathrm{~min}$. Therefore, in the absence of any noticeable forgetting, we believe that memory decay is an unlikely mechanism driving subjects' performance.

As mentioned above, the other possible mechanism involved in successful memory retrieval is familiarity. Familiarity is a recognition process that provides a temporal cue for knowing which food item will be available without actually recollecting the past event (Kort et al. 2005). However, we believe that familiarity cannot be the mechanism underlying apes' choices. Subjects remained in the cage with the platform in sight in Experiment 1 and 2 during both trials. Additionally, in Experiment 2 the temporal pattern of exposure to the platforms was identical for the two types of trials. Therefore, apes could not have learnt to choose the frozen juice when the platforms were relatively familiar and the grape when the platforms were unfamiliar because apes were exposed to both platforms for the same duration of time. However, note that our data do not allow us to conclude that apes are actually recalling the baiting episode.

A number of theorists (Suddendorf and Corballis 1997, 2007; Tulving 2002, 2005) has argued that some of the studies on www-memory in nonhuman animals could be explained as instinctive or learning predispositions (i.e. caching behaviour in scrub jays). However, we suggest that it is unlikely that the apes' behaviour was based on a similar instinctual predisposition to recover hidden caches or reject frozen juice after longer RI since none of the aspects is part of the ecology where apes evolved.

One of the aspects that has received no attention in the study of the episodic memory in nonhuman animals is the ontogeny of the components of this memory system. Suddendorf and Busby (2003) argued that this information could highlight the existence of developmental parallels between different species, supporting the argument that this capacity is present in other species. The current study helps to provide these much needed developmental data. The performance of the members of the genus Pan (chimpanzees and bonobos) followed an inverted U-shaped distribution. We consider that this result is intriguing for two reasons. First, this age-dependent performance is similar to the one reported for chimpanzees in the mirror self-recognition (MSR) task (Povinelli et al. 1993), a task typically used to assess the development of self-concept in human infants (Amsterdam 1972; Lewis and Brooks-Gunn 1979; Nielsen and Dissanayake 2004). Although other studies (e.g., Bard et al., 2006) have reported evidence of MSR at an earlier age than Povinelli et al. (1993), we think that the latter study offers us a better point of comparison for our data because of its broad age distribution, larger sample size and the inclusion of some additional control conditions that are absent in other studies. Nevertheless, the relation between www-memory and self-recognition in Pan should be interpreted with caution due to the existing discrepancies between studies regarding the age of emergence of MSR in chimpanzees.

Second, an inverted U-shape distribution is a feature of episodic memory in humans. One could speculate that the similarity between the human data and the ape data might indicate that humans, chimpanzees and bonobos share some information encoding and storage mechanisms. Further research will be necessary to put this idea to test with additional tasks and larger samples of individuals. It is 
precisely our reduced sample size that does not allow us to carry out more fine-grained analyses in order to tell apart differences between the great ape species included in this study and the developmental patterns in this memory system. A possible explanation for our results could be that the orangutans perform worse at the www-memory task, but it might also be plausible that the orangutans that we tested are too young or too old that their performance is consistent with what is expected from a developmental perspective. Besides documenting the developmental trajectories of memory systems in various species, future studies should also attempt to operationalize those aspects of the episodic memory system in humans to bring them under rigorous empirical scrutiny in nonverbal organisms. It will be equally important to determine whether apes process the 'when' component as either elapsed time or as mental time travel to a past event.

Acknowledgments This work was supported in part by grant HA2005-0010 (Acciones Integradas between Spain and Germany) from the Spanish Ministry of Education and Science to F. Colmenares and a FPI studentship from the Spanish Ministry of Education and Science to Gema Martin-Ordas. The experiments comply with the current German laws where they were performed.

Open Access This article is distributed under the terms of the Creative Commons Attribution Noncommercial License which permits any noncommercial use, distribution, and reproduction in any medium, provided the original author(s) and source are credited.

\section{References}

Adams DC, Anthony CD (1996) Using randomization techniques to analyze behavioural data. Anim Behav 51:733-738

Amsterdam B (1972) Mirror self-image reactions before age two. Dev Psychobiol 5:297-305

Babb SJ, Crystal JD (2005) Discrimination of what, when and where: implications for episodic-like memory in the rat. Learn Motiv 36:177-189

Babb SJ, Crystal JD (2006) Discrimination of what, when and where is not based on the time of the day. Learn Behav 34:124-130

Baddeley A (2000) The episodic buffer: a new component of working memory? Trends Cogn Sci 4:417-423

Bard K, Todd BK, Bernier C, Love J, Leavens DA (2006) Self-awareness in human and chimpanzee infants: what is measured and what is meant by the mark and mirror test. Infancy 9:191-219

Bialystok E, Craik FIM (2006) Lifespan cognition: mechanisms of change. Oxford University Press, New York

Bird LR, Roberts WA, Abroms B, Kit KA, Crupi C (2003) Spatial memory for food hidden by rats (Rattus norvegicus) on the radial maze: studies of memory for what, where and when. J Comp Psychol 117:176-187

Clayton NS, Dickinson A (1998) Episodic-like memory during cache recovery by scrub jays. Nature 395:272-274

Clayton NS, Dickinson A (1999) Scrub jays (Aphelocoma coerulescens) remember the relative time of caching as well as the location and content of their caches. J Comp Psychol 113:403-416
Clayton NS, Yu KS, Dickinson A (2001) Scrub-jays (Aphelocoma coerulescens) form integrated memories of the multiple features of caching episodes. J Exp Psychol Anim Behav Proc 27:17-29

Clayton NS, Bussey TJ, Dickinson A (2003a) Can animals recall the past and plan for the future? Nat Rev Neurosci 4:685-691

Clayton NS, Yu KS, Dickinson A (2003b) Interacting cache memories: evidence for flexible memory use by western scrubjays (Aphelocoma coerulescens). J Exp Psychol Anim Behav Proc 29:14-22

Craik FIM, Salthouse TA (2000) Handbook of aging and cognition, 2nd edn. Lawrence Erlbaum Associates, Hillsdale

Craik FIM, Salthouse TA (2008) Handbook of aging and cognition, 3rd edn. Lawrence Erlbaum Associates, Hillsdale

Crystal JD (2009) Elements of episodic-like memory in animal models. Behav Process 80:269-277

Dere E, Huston JP, De Souza Silva MA (2005) Integrated memory for objects, places and temporal order: evidence for episodic-like memory in mice. Neurobiol Learn Mem 84:214-221

Dufour V, Sterck EHM (2008) Chimpanzees fail to plan in an exchange task but succeed in a tool-using procedure. Behav Process 79:19-27

Eacott MJ, Easton A, Zinkivsky A (2005) Recollection in an episodiclike memory task in the rat. Learn Mem 12:221-223

Ergorul C, Eichenbaum H (2004) The hippocampus and memory for "what", "where", and "when". Learn Mem 11:397-405

Ferkin MH, Combs A, delBarco-Trillo J, Pierce AA, Franklin S (2008) Meadow voles, Microtus pennsylvanicus, have the capacity to recall the "what", "where" and "when" of a single past event. Anim Cogn 11:147-159

Friedman WJ (1993) Memory for the time of past events. Psychol Bull 113:44-66

Friedman WJ (2001) Memory processes underlying humans' chronological sense of the past. In: Hoerl C, McCormack T (eds) Time and memory: issues in philosophy and psychology. Oxford University Press, Oxford, pp 139-167

Friedman WJ (2004) Time in autobiographical memory. Soc Cogn 22:605-621

Friedman WJ (2007) The meaning of "time" in episodic memory and mental time travel. Behav Brain Sci 30:323

Haccou P, Meelis E (1994) Statistical analyses of behavioural data. Oxford University Press, Oxford

Hampton RR, Hampstead BM, Murray EA (2005) Rhesus monkeys (Macaca mulatta) demonstrate robust memory for what and where, but not for when, in an open-field test of memory. Learn Motiv 36:245-259

Henderson J, Hurly TA, Bateson M, Healy SD (2006) Timing in free living rufous humming birds, Selasphorus rufus. Curr Biol $16: 512-515$

Hoffman ML, Beran, MJ, Washburn DA (2009) Memory for "what", "where" and "when" information in rhesus monkeys (Macaca mulatta). J Exp Psychol Anim Behav Proc (in press)

Kart-Teke E, De Souza Silva MA, Huston JP, Dere E (2006) Wistar rats show episodic-like memory for unique experiences. Neurobiol Learn Mem 85:173-182

Kort SR, Dickinson A, Clayton NS (2005) Retrospective cognition by food-caching western scrub-jays. Learn Motiv 36:159-176

Lewis M, Brooks-Gunn J (1979) Social cognition and the acquisition of self. Plenum Press, New York

Manly BFJ (1997) Randomization, bootstrap and Monte Carlo methods in biology. Chapman and Hall, New York

McKenzie TLB, Bird LR, Roberts WA (2005) The effects of cache modification on food caching and retrieval bahvior by rats. Learn Motiv 36:260-278

Menzel E (2005) Progress in the study of chimpanzee recall and episodic memory. In: Terrace HS, Metcalfe J (eds) The missing link in cognition. Oxford University Press, Oxford, pp 188-224 
Miyashita Y (2004) Cognitive memory: cellular and network machineries and their top-down control. Science 306:435-440

Morris RGM, Frey U (1997) Hippocampal synaptic plasticity: role in spatial learning or the automatic recording of attended information? Philos Trans R Soc Lond B Biol Sci 352:1489-1503

Mulcahy NJ, Call J (2006) Apes save tools for future use. Science 312:1038-1040

Nielsen M, Dissanayake C (2004) Pretend play, mirror self-recognition and imitation: a longitudinal investigation through the second year. Infant Behav Dev 27:342-365

Osvath M, Osvath H (2008) Chimpanzee (Pan troglodytes) and orangutan (Pongo abelii) forethought: self-control and preexperience in the face of future tool use. Anim Cogn 11:661-674

Povinelli DJ, Rulf AB, Landau KR, Bierschwale DT (1993) Selfrecognition in Chimpanzees (Pan troglodytes): distribution, ontogeny, and patterns of emergence. J Comp Psych 107:347-372

R Development Core Team (2007) R: a language and environment for statistical computing. R Foundation for Statistical Computing, Vienna, Austria

Roberts WA, Feeney MC, MacPherson K, Petter M, McMillan N, Musolino E (2008) Episodic-like memory in rats: is it based on when or how long ago? Science 320:113-115

Salwiczek LH, Dickinson A, Clayton NS (2008) What do animals remember about their past? In: Menzel R (ed) Learning theory and behavior. Volume 1 of learning and memory: a comprehensive reference, 4 vols (Byrne J editor). Elsevier, Oxford, pp 441-460

Schwartz BL (2005) Do humans primates have episodic memory? In: Terrace HS, Metcalfe J (eds) The missing link in cognition. Oxford University Press, Oxford, pp 225-241

Schwartz BL, Colon MR, Sanchez IC, Rodriguez IA, Evans S (2002) Single-trial learning of "what" and "who" information in a gorilla (Gorilla gorilla gorilla): implications for episodic memory. Anim Cogn 5:85-90

Schwartz BL, Hoffman ML, Evans S (2005) Episodic-like memory in a gorilla: a review and new findings. Learn Motiv 36:226-244

Skov-Rackette SI, Miller NY, Shettleworth SJ (2006) What-wherewhen memory in pigeons. J Exp Psychol Anim Behav 32:345-358
Squire LR (1992) Declarative and nondeclarative memory: multiple brain systems supporting learning and memory. J Cogn Neurosci $4: 232-243$

Suddendorf T (2006) Foresight and evolution of the human mind. Science 312:1006-1007

Suddendorf T, Busby J (2003) Like it or not? The mental time travel debate. Trends Cogn Sci 7:437-438

Suddendorf T, Corballis MC (1997) Mental time travel and the evolution of the human mind. Genet Soc Gen Psychol Monogr 123:133-167

Suddendorf T, Corballis MC (2007) The evolution of foresight: What is mental time travel, and is it unique to humans? Behav Brain Sci 30:299-351

Suddendorf T, Corballis MC, Collier-Baker E (2009) How great is great ape foresight? Anim Cogn. DOI 10.1007/s10071-0090253-9

Tulving E (1972) Episodic and semantic memory. In: Tulving E, Donaldson W (eds) Organization of memory. Academic Press, New York

Tulving E (1983) Elements of episodic memory. Oxford University Press

Tulving E (2002) Episodic memory: from mind to brain. Annu Rev Psychol 53:1-25

Tulving E (2005) Episodic memory and autonoesis: uniquely human? In: Terrace HS, Metcalfe J (eds) The missing link in cognition. Oxford University Press, Oxford, pp 3-56

Tulving E, Craik F (2000) The Oxford handbook of memory. Oxford University Press, Oxford

Zentall TR, Clement TS, Bhatt RS, Allen J (2001) Episodic-like memory in pigeons. Psychon Bull Rev 8:685-690

Zhou W, Crystal JD (2009) Evidence for remembering when events occurred in a rodent model of episodic memory. Proc Natl Acad Sci USA 106:9525-9529

Zinkivskay A, Nazir F, Smulders TV (2009) What-where-when memory in magpies (Pica pica). Anim Cogn 12:119-125 